\title{
NOVEL ALGORITHMS FOR TRACKING MULTIPLE TARGETS
}

Sheng-Yun Hou

Department of Electrical Engineering, National Taiwan Ocean University, Keelung, Taiwan, R.O.C Department of Electronic Engineering, Hwa Hsia Institute of Technology, Chung Ho, Taipei, Taiwan, R.O.C

Hsien-Sen Hung

Department of Electrical Engineering, National Taiwan Ocean University, Keelung, Taiwan, R.O.C., b0221@mail.ntou.edu.tw

Shun-Hsyung Chang

Department of Microelectronic Engineering, National Kaohsiung Marine University, Kaohsiung, Taiwan, R.O.C

Jeng-Cheng Liu

Department of Electrical Engineering, National Taiwan Ocean University, Keelung, Taiwan, R.O.C.

Follow this and additional works at: https://jmstt.ntou.edu.tw/journal

Part of the Electrical and Computer Engineering Commons

\section{Recommended Citation}

Hou, Sheng-Yun; Hung, Hsien-Sen; Chang, Shun-Hsyung; and Liu, Jeng-Cheng (2010) "NOVEL ALGORITHMS FOR TRACKING MULTIPLE TARGETS," Journal of Marine Science and Technology. Vol. 18: Iss. 2, Article 12.

DOI: $10.51400 / 2709-6998.2325$

Available at: https://jmstt.ntou.edu.tw/journal/vol18/iss2/12

This Research Article is brought to you for free and open access by Journal of Marine Science and Technology. It has been accepted for inclusion in Journal of Marine Science and Technology by an authorized editor of Journal of Marine Science and Technology. 


\section{NOVEL ALGORITHMS FOR TRACKING MULTIPLE TARGETS}

\section{Acknowledgements}

This work was supported in part by National Science Council of TAIWAN under contracts NSC 98-2221-E-019-066- and NSC 96-2221-E-022-016-. The authors would like to thank unknown reviewers for invaluable comments and suggestions. 


\title{
NOVEL ALGORITHMS FOR TRACKING MULTIPLE TARGETS
}

\author{
Sheng-Yun Hou***, Hsien-Sen Hung*, Shun-Hsyung Chang***, and Jeng-Cheng Liu*
}

Key words: angle tracking, bearing estimation, Kalman filter, extended Kalman filter, particle filter, direction of arrival, multitarget tracking.

\begin{abstract}
In this paper, two novel angle tracking algorithms are proposed for tracking multiple targets using an array of sensors with known locations. First, we present an extended Kalman particle filter (EKPF) which is capable of determining the direction-of-arrival (DOA) angles using a single snapshot of data during the interval between each time step. The proposed EKPF algorithm combines particle filtering with the extended Kalman filter (EKF) in order to prevent sample impoverishment during its resampling process. Next, we present a robust Kalman filter (RKF) tracking algorithm intended to improve tracking success rates of other existing algorithms for the case of multiple snapshots of data within each time increment. In the proposed RKF algorithm, a robust decision mechanism is proposed and incorporated into the Kalman filter (KF), leading to a much better tracking success rate. Because KF (or EKF) is able to offer the predictability of DOA angles, the proposed EKPF and RKF algorithms can avoid the data association problem that usually occurs in multitarget tracking. The effectiveness of the proposed algorithms are demonstrated via computer simulations in scenarios involving targets with crossing trajectories.
\end{abstract}

\section{INTRODUCTION}

Tracking multiple targets based on measurements of sensor array is an important research topic in the fields of sonar, radar, air traffic control, remote sensing as well as wireless communications. Various target angle tracking algorithms have been proposed and reported in the literature for multiple narrow-

\footnotetext{
Paper submitted 10/03/08; revised 05/09/09; accepted 06/08/09. Author for correspondence: Hsien-Sen Hung (e-mail: b0221@mail.ntou.edu.tw).

*Department of Electrical Engineering, National Taiwan Ocean University, Keelung, Taiwan, R.O.C.

**Department of Electronic Engineering, Hwa Hsia Institute of Technology, Chung Ho, Taipei, Taiwan, R.O.C.

***Department of Microelectronic Engineering, National Kaohsiung Marine University, Kaohsiung, Taiwan, R.O.C.
}

band targets $[5-8,10,11,14-16]$. Yang and Kaveh proposed an iterative adaptive eigen-subspace method in conjunction with the MUSIC algorithm to track the direction-of-arrival (DOA) angles of multiple targets [16]. Due to the data association problem caused by multi-target tracking, the adaptive MUSIC method fails to track targets when they are moving closer. Although the method proposed by Sword et al. [14] can avoid the data association problem, errors are accumulated in each iteration making it unable to track targets that are mutually close. Due to the nature of prediction-correction filtering process, Kalman filter (KF) can reduce estimation errors and avoid the data association problem when applied to angle tracking, as stated in several references $[5,7,8]$. Rao et al. [8] proposed to estimate DOA angles of targets using the maximum likelihood method and feeding the results to the KF. However, it assumes that the signal powers of the targets are all different, making the algorithm impractical. Javier and Sylvie [5] suggested to estimate target angles using the projection approximation subspace tracking algorithm with deflation (PASTd) [15] and a Newton-type method (for MUSIC spectrum) for the use in the KF. It has lower computational load and better tracking performance than Rao's algorithm, but still exhibits poor tracking success rate at low signal-to-noise ratios (SNRs). Park et al. [7] proposed an approach which utilizes predicted angles obtained from Sword's method. The approach also uses the constrained least-squares criterion to confine the dynamic range of angles. The choice of relevant parameters is empirical and is not suitable for various scenarios of different moving speeds and signal-to-noise ratios. Besides, tracking performance degrades seriously with an increasing number of crossing targets. Later on, in order to improve Park's method, Ryu et al. $[10,11]$ suggested to obtain the angle innovations of the targets from a signal subspace, instead of the sensor output covariance matrix, via PAST algorithm [15]. Chang et al. [3] modified Park's algorithm by incorporating a spatial smoothing technique to overcome multipath interference, and also coherent signal-subspace processing for tracking wideband targets. All of the above algorithms are based on the sample covariance matrix or signal subspace made with multiple snapshots of data from a sensor array. However, they all fail to track multiple targets when only a single snapshot measurement is available between two consecutive time steps during the tracking process, because DOA estimation using subspace-based approach re- 
quires sample covariance matrix or signal subspace with a rank of more than one.

For the case of a single snapshot measurement within each time increment, tracking multiple targets becomes feasible if the sensor array output is directly used as the measurement data in the extended Kalman filter (EKF) [6]. The EKF is an approximate nonlinear state estimation technique with firstorder linearization accuracy, and is suitable for the tracking problem since the measurement model is nonlinear in terms of the angles (states) to be estimated. The algorithm proposed by Kong and Chun [6] exhibits low tracking success rate when targets approach near the points of intersection. The reason for this weakness is the EKF can be difficult to tune and often gives unreliable estimates if the system nonlinearities are severe. Unlike the EKF, the particle filter from Metropolis and Wiener is a completely nonlinear state estimator [4]. It can provide higher tracking performance at the price of an increased level of computational efforts. However, the particle filter has the potential problem of sample impoverishment during its resampling process. This problem will be made worse if the measurements are not consistent with the process model [13].

In this paper, we propose two novel tracking algorithms. The first one, named as EKPF (Extended Kalman Particle Filter) tracking algorithm, is suitable for the case of a single snapshot measurement within each time increment. EKPF combines particle filtering with the EKF to prevent sample impoverishment. It leads to remarkable performance improvement over the particle filter alone and the EKF, as demonstrated by simulation results. The second one, named as RKF (Robust Kalman Filter) tracking algorithm, is intended to improve the tracking success rates of other existing algorithms for the case of multiple snapshot measurements within each time increment. RKF modifies Javier's algorithm by replacing the PASTd algorithm with the Sliding Window Orthonormal PAST (SW-OPAST) algorithm [1], and incorporating a robust decision mechanism into KF. SW-OPAST can provide faster tracking response than PASTd during the process of obtaining orthonormal basis vectors of noise subspace for the subsequent MUSIC algorithm. Our proposed robust decision mechanism is helpful in preventing large errors occurring when target locations are mutually close, thus significantly improving the tracking success rate especially at low SNRs as demonstrated by simulation results.

\section{SYSTEM MODEL}

We consider $M$ targets moving in a plane containing an array of $L$ sensors. The sensor positions are assumed to be known, and we take them to be placed uniformly on a line with spacing of $d$ between two adjacent sensors, measured in the unit of wavelength $\lambda$. The motion of the targets is assumed to be at constant angular speed in the presence of Gaussian disturbance, and is observed every $T$ seconds. Let $\theta_{m}(t) \in$ $\left[-\frac{\pi}{2}, \frac{\pi}{2}\right]$, measured clockwise with respect to $y$ axis, denote the DOA angle of the $m$ th target at time $t$. Assuming that these targets are located in the far field and their radiated signals are narrowband with a common angular frequency $\omega_{0}$, the output of the $l$ th isotropic sensor at time $t$ is then

$$
r_{l}(t)=\sum_{m=1}^{M} e^{-j \omega_{o} \tau_{l m}} s_{m}(t)+n_{l}(t)
$$

where $s_{m}(t) \in R$ is the signal transmitted by the $m$ th target at time $t, n_{l}(t)$ is a complex Gaussian white noise with zero mean and variance $\sigma_{n}^{2}$, which is uncorrelated with the target signals, and $\tau_{l m}$ is the difference in time delays of the $m$ th target reaching the first (reference) sensor and the $l$ th sensor. By using vector-matrix representation, the output of the sensor array is given by

$$
\mathbf{r}(t)=\mathbf{A}[\boldsymbol{\theta}(t)] \mathbf{s}(t)+\mathbf{n}(t)
$$

where $\mathbf{r}(t)=\left[r_{1}(t), \ldots, r_{L}(t)\right]^{T}, \mathbf{s}(t)=\left[s_{1}(t), \ldots, s_{M}(t)\right]^{T}, \mathbf{n}(t)=$ $\left[n_{1}(t), \ldots, n_{L}(t)\right]^{T}$ are the output data, target signal, and noise vectors, respectively. $\boldsymbol{\theta}(t)=\left[\theta_{1}(t), \theta_{2}(t), \ldots, \theta_{M}(t)\right]^{T}$ is the target DOA vector and $\mathbf{A}[\boldsymbol{\theta}(t)]$ is the array direction matrix with the direction vector of the $m$ th target (the $m$ th column vector)

$$
\mathbf{a}_{m}=\left[1, e^{-j \frac{2 \pi}{\lambda} d \sin \theta_{m}}, \ldots, e^{-j \frac{2 \pi}{\lambda}(L-1) d \sin \theta_{m}}\right]^{T}
$$

Suppose there are $K$ measurements (snapshots) that are taken for each increment $T$, and the time increment is sufficiently small allowing us to approximate the target as stationary. The tracking problem is aimed at estimating $\boldsymbol{\theta}(t), t=T$, $2 T, \ldots$ from $K$ snapshots of array data measured within each time increment $T$.

\section{THE PROPOSED TRACKING ALGORITHMS}

\section{The EKPF Tracking Algorithm}

For the case of a single snapshot measurement within each time increment, the EKPF algorithm is proposed and stated as follows. First, we describe the discrete-time state (process) model for the target motion described in the previous section. For each time index $k$, we define the state vector for the $m$ th target as $\mathbf{x}_{m}(k)=\left[\begin{array}{ll}\theta_{m}(k) & \dot{\theta}_{m}(k)\end{array}\right]^{T}$, consisting of its DOA angle and angular speed. The target motion can lead to the process equation [8]

$$
\begin{aligned}
\mathbf{x}_{m}(k+1) & =\mathbf{F} \mathbf{x}_{m}(k)+\mathbf{w}_{m}(k) \\
\mathbf{F} & =\left[\begin{array}{cc}
1 & T \\
0 & 1
\end{array}\right]
\end{aligned}
$$


where $\mathbf{w}_{m}(k)$ is the process noise vector and is assumed to be Gaussian distributed with zero mean and covariance

$$
\mathbf{Q}_{m}=\sigma_{w}^{2}\left[\begin{array}{cc}
\frac{T^{3}}{3} & \frac{T^{2}}{2} \\
\frac{T^{2}}{2} & T
\end{array}\right]
$$

Assume that the motion of each target is mutually independent. By defining the composite state vector as $\mathbf{x}(k)=$ $\left[\mathbf{x}_{1}^{T}(k), \ldots, \mathbf{x}_{M}^{T}(k)\right]^{T}$, the system dynamics is governed by the process model

$$
\begin{gathered}
\mathbf{x}(k+1)=\overline{\mathbf{F}} \mathbf{x}(k)+\mathbf{w}(k) \\
\overline{\mathbf{F}}=\left[\begin{array}{ccccc}
1 & T & & & 0 \\
0 & 1 & & & \\
& & \ddots & & \\
& & & 1 & T \\
0 & & & 0 & 1
\end{array}\right]
\end{gathered}
$$

The process noise vector $\mathbf{w}(k)$ reflects the random modeling error, which is Gaussian distributed with zero mean vector and covariance

$$
\overline{\mathbf{Q}}=\sigma_{w}^{2}\left[\begin{array}{cccccc}
\frac{T^{3}}{3} & \frac{T^{2}}{2} & & & & \\
\frac{T^{2}}{2} & T & & & & \\
& & \ddots & & & \\
& & & & \frac{T^{3}}{3} & \frac{T^{2}}{2} \\
0 & & & \frac{T^{2}}{2} & T
\end{array}\right]
$$

The matrices $\overline{\mathbf{F}}$ and $\overline{\mathbf{Q}}$ are all block diagonal. Although the process equation is a linear model, the measurement model of (2) is a vector nonlinear function of the target DOA angles (and thus, of the target state vectors as well), which can be restated as

$$
\mathbf{r}(k) \triangleq \mathbf{h}(\mathbf{x}(k), \mathbf{s}(k), \mathbf{n}(k))=\mathbf{A}(\mathbf{x}(k)) \mathbf{s}(k)+\mathbf{n}(k)
$$

where $\mathbf{n}(k)$ is complex Gaussian noise process with the known covariance $\sigma_{n}^{2} \mathbf{I}$, and is assumed to be uncorrelated with the process noise $\mathbf{w}(k)$.
In this section, we propose to use the EKF [13] for the nonlinear tracking system to obtain high performance. The proposed EKPF tracking algorithm is formed by combining the particle filter with the EKF in order to prevent sample impoverishment in the resampling process of the particle filter. Since the particle filter was invented to numerically implement the Bayesian state estimator for nonlinear/nonGaussian systems [4], it is anticipated that the proposed tracking algorithm can achieve superior performance even if the measurement noise is not Gaussian. In this paper, we make use of the particle filter described in [9], because the main idea is intuitive and straightforward.

The proposed tracking algorithm for the case of a single snapshot measurement is stated as follows.

- Initially (at $k=0$ ), the target DOA angles, $\left\{\hat{\theta}_{m}(-1)\right\}$ and $\left\{\hat{\theta}_{m}(0)\right\}$ at two successive time instants, $k=-1$ and $k=0$, are assumed to be available, which can be estimated by any kind of angle estimation algorithm (for instance the MUSIC algorithm [12]). Assuming that the state vector is Gaussian distributed, we then randomly generate $N$ state vectors, based on the Gaussian probability density function (pdf) with the mean vector $\mathbf{x}(0 \mid 0)=\left[\hat{\theta}_{1}(0), \quad\left(\hat{\theta}_{1}(0)-\hat{\theta}_{1}(-1)\right) /\right.$ $\left.T, \ldots, \hat{\theta}_{M}(0),\left(\hat{\theta}_{M}(0)-\hat{\theta}_{M}(-1)\right) / T\right]^{T}$ and the covariance

$$
\mathbf{P}(0 \mid 0)=\sigma_{v}^{2}\left[\begin{array}{cccccc}
1 & \frac{1}{T} & & & & \\
\frac{1}{T} & \frac{2}{T^{2}} & & & & 0 \\
& & \ddots & \ddots & & \\
& & & & 1 & \frac{1}{T} \\
0 & & & \frac{1}{T} & \frac{2}{T^{2}}
\end{array}\right]
$$

These state vectors are called particles and are denoted $\mathbf{x}^{(i)}(0 \mid 0)$. Their covariance matrices are denoted $\mathbf{P}^{(i)}(0 \mid 0)=$ $\mathbf{P}(0 \mid 0)(i=1, \ldots, N)$. The parameter $N$ is chosen by the user as a trade-off between computational effort and estimation accuracy.

- For $k=1,2, \ldots$, do the following.

(a) Perform the time propagation step to obtain a priori particles $\mathbf{x}^{(i)}(k \mid k-1)$ and covariances $\mathbf{P}^{(i)}(k \mid k-1)$ using

$$
\begin{gathered}
\mathbf{x}^{(i)}(k \mid k-1)=\overline{\mathbf{F}} \mathbf{x}^{(i)}(k-1 \mid k-1)+\mathbf{w}(k) \\
\mathbf{P}^{(i)}(k \mid k-1)=\overline{\mathbf{F}} \mathbf{P}^{(i)}(k-1 \mid k-1) \overline{\mathbf{F}}^{T}+\overline{\mathbf{Q}}
\end{gathered}
$$


Thus, the predicted array direction matrix, $\mathbf{A}^{(i)}(k \mid k-1)$ can be obtained from (6) using $\mathbf{x}^{(i)}(k \mid k-1)$. The complex measurement vector $\mathbf{r}(k)$ and the array direction matrix $\mathbf{A}^{(i)}(k \mid k-1)$ can be expressed as the composite real vector and matrix, i.e.

$$
\overline{\mathbf{r}}(k)=\left[\begin{array}{c}
\operatorname{real}(\mathbf{r}(k)) \\
\operatorname{imag}(\mathbf{r}(k))
\end{array}\right], \overline{\mathbf{A}}^{(i)}(k \mid k-1)=\left[\begin{array}{c}
\operatorname{real}\left(\mathbf{A}^{(i)}(k \mid k-1)\right) \\
\operatorname{imag}\left(\mathbf{A}^{(i)}(k \mid k-1)\right)
\end{array}\right]
$$

By applying the maximum likelihood principle to (6), we can obtain the target signal vectors $\mathbf{s}^{(i)}(k)=\left[s_{1}^{(i)}(k)\right.$,

$\left.\cdots, s_{M}^{(i)}(k)\right]^{T}$ as

$$
\mathbf{s}^{(i)}(k)=\left[\overline{\mathbf{A}}^{(i)^{H}}(k \mid k-1) \overline{\mathbf{A}}^{(i)}(k \mid k-1)\right]^{-1} \overline{\mathbf{A}}^{(i)^{H}}(k \mid k-1) \overline{\mathbf{r}}(k)
$$

Therefore, the composite real predicted sensor array output vector becomes

$$
\overline{\mathbf{r}}^{(i)}(k \mid k-1)=\overline{\mathbf{A}}^{(i)}(k \mid k-1) \mathbf{s}^{(i)}(k)
$$

(b) Update the a priori particles and covariances to obtain $a$ posteriori particles and covariances:

The partial derivative matrix of the measurement model (6) is given by

$$
\mathbf{H}^{(i)}(k)=\left.\frac{\partial \mathbf{h}}{\partial \mathbf{x}}\right|_{\mathbf{x}=\mathbf{x}^{(i)}(k \mid k-1)}=\left.\left[\mathbf{H}_{1}(k), \ldots, \mathbf{H}_{M}(k)\right]\right|_{\mathbf{x}=\mathbf{x}^{(i)}(k \mid k-1)}
$$

By augmenting the real and imaginary parts of each complex matrix $\mathbf{H}_{m}(k)$, we have the composite real matrix of dimension $2 L \times 2 M$

$$
\overline{\mathbf{H}}^{(i)}(k)=\left[\begin{array}{c}
\operatorname{real}\left(\mathbf{H}_{1}(k), \ldots, \mathbf{H}_{M}(k)\right) \\
\operatorname{imag}\left(\mathbf{H}_{1}(k), \ldots, \mathbf{H}_{M}(k)\right)
\end{array}\right]_{\mathbf{x}=\mathbf{x}}^{(i)}(k \mid k-1)
$$

which can be expressed as

$$
\overline{\mathbf{H}}^{(i)}(k)=\left[\begin{array}{ccccc}
0 & 0 & \cdots & 0 & 0 \\
p_{1,1} & 0 & \cdots & p_{M, 1} & 0 \\
\vdots & \vdots & \ddots & \vdots & \vdots \\
p_{1, L-1} & 0 & \cdots & p_{M, L-1} & 0 \\
0 & 0 & \cdots & 0 & 0 \\
c_{1,1} & 0 & \cdots & c_{M, 1} & 0 \\
\vdots & \vdots & \ddots & \vdots & \vdots \\
c_{1, L-1} & 0 & \cdots & c_{M, L-1} & 0
\end{array}\right]_{\mathbf{x}=\mathbf{x}^{(i)}(k \mid k-1)}
$$

where $p_{m, b}=-\sin \left(\pi b \sin \left(\theta_{m}(k)\right)\right) \cos \left(\theta_{m}(k)\right) s_{m}(k), c_{m, b}=-\cos$ $\left(\pi b \sin \left(\theta_{m}(k)\right)\right) \cos \left(\theta_{m}(k)\right) s_{m}(k), m=1, \ldots, M, b=1, \ldots, L-1$.
Thus, the Kalman gain matrices $\mathbf{K}^{(i)}$ are given by

$$
\mathbf{K}^{(i)}(k)=\mathbf{P}^{(i)}(k \mid k-1) \overline{\mathbf{H}}^{(i) T}(k)\left[\overline{\mathbf{H}}^{(i)}(k) \mathbf{P}^{(i)}(k \mid k-1) \overline{\mathbf{H}}^{(i) T}(k)+\sigma_{n}^{2} \mathbf{I}^{-1}\right.
$$

and a posteriori particles are then updated according to

$$
\mathbf{x}^{(i)}(k \mid k)=\mathbf{x}^{(i)}(k \mid k-1)+\mathbf{K}^{(i)}(k)\left[\overline{\mathbf{r}}(k)-\overline{\mathbf{r}}^{(i)}(k \mid k-1)\right]
$$

The covariances of a posteriori particles are given by

$$
\mathbf{P}^{(i)}(k \mid k)=\left(\mathbf{I}-\mathbf{K}^{(i)}(k) \overline{\mathbf{H}}^{(i)}(k)\right) \mathbf{P}^{(i)}(k \mid k-1)
$$

(c) Compute the relative likelihood $q^{(i)}$ of each a posteriori particle conditioned on the measurement $\mathbf{r}(k)$. This is done by evaluating the Gaussian pdf $p\left(\mathbf{r}(k) \mid \mathbf{x}^{(i)}(k \mid k)\right.$, $\left.\mathbf{s}^{(i)}(k)\right)$, which is the Gaussian distributed with mean vector $\overline{\mathbf{A}}^{(i)}(k \mid k) \mathbf{s}^{(i)}(k)$ and covariance $\sigma_{n}^{2} \mathbf{I}$, wherein $\overline{\mathbf{A}}^{(i)}(k \mid k)$ is obtained using a posteriori particles. Namely,

$$
q^{(i)} \sim \exp \left[-\frac{\left(\overline{\mathbf{r}}(k)-\overline{\mathbf{A}}^{(i)}(k \mid k) \mathbf{s}^{(i)}(k)\right)^{T}\left(\overline{\mathbf{r}}(k)-\overline{\mathbf{A}}^{(i)}(k \mid k) \mathbf{s}^{(i)}(k)\right)}{2 \sigma_{n}^{2}}\right]
$$

We then normalize the relative likelihoods $q^{(i)}$ as

$$
q^{(i)}=\frac{q^{(i)}}{\sum_{j=1}^{N} q^{(j)}}
$$

This ensures that the sum of all the likelihoods is equal to one.

(d) Refine the set of a posteriori particles and covariances based on the normalized relative likelihoods $q^{(i)}$. This can be done several different ways. Here, we use the resampling way of [9], which is described as follows. For $i=$ $1, \ldots, N$, perform the following two steps: First, generate a random number $\gamma$ that is uniformly distributed on $[0,1]$. Second, accumulate the likelihoods $q^{(i)}$ into a sum, one at time, until the accumulated sum is greater than $\gamma$. The new particle $\mathbf{x}^{(i)}(k \mid k)$ is then set equal to the old particle $\mathbf{x}^{(j)}(k \mid k-1)(i, j=1, \ldots, N)$.

(e) Compute the expected value of posterior pdf $p(\mathbf{x}(k) \mid \mathbf{r}(k))$ by approximating it as the algebraic mean of the a posteriori particles

$$
E(\mathbf{x}(k) \mid \mathbf{r}(k)) \approx \frac{1}{N} \sum_{i=1}^{N} \mathbf{x}^{(i)}(k \mid k)
$$


Repeat the steps of (a)-(e) until $K$ measurements are used, then average over these resultant expected values to obtain a refined state vector.

\section{The RKF Tracking Algorithm}

For the case of multiple snapshot measurements within each time increment, the RKF algorithm is presented. Traditionally, the signal subspace $\mathbf{W}$ is obtained by an eigendecomposition of the sample covariance matrix of the sensor array data, which involves a heavy computational load (at least $O\left(L^{2} K\right)$ operations for the computation of sample covariance matrix) and thus has limited use for tracking. Here we suggest to estimate $\mathbf{W}$ via the SW-OPAST algorithm, which only requires $O(L M K)$ operations [1]. The SW-OPAST algorithm uses the recursive least squares (RLS) principle with a sliding window to obtain faster response in tracking the approximate signal subspace. As such, the subspace $\mathbf{W}(k)$ can be tracked to offer the orthonormal basis vectors for the noise subspace required by the MUSIC algorithm in an adaptive manner as the time $k$ evolves. In addition, a robust decision mechanism is proposed to be incorporated into the KF procedure. It is aimed to prevent large errors which often occur when the target locations are mutually close, thus potentially improving the tracking success rate particularly at low SNRs. The predictability characteristic of KF can avoid the data association problem for tracking multiple targets.

Suppose $\tilde{\mathbf{x}}_{m}(k)=\left[\begin{array}{lll}\theta_{m}(k) & \dot{\theta}_{m}(k) & \ddot{\theta}_{m}(k)\end{array}\right]^{T}$ denotes the state vector for the $m$ th source at time $k$. We model the dynamics and measurement equations of the $m$ th source by $[2,5]$

$$
\begin{gathered}
\tilde{\mathbf{x}}_{m}(k+1)=\tilde{\mathbf{F}} \tilde{\mathbf{x}}_{m}(k)+\mathbf{w}_{m}(k) \\
\hat{\theta}_{m}(k)=\mathbf{h} \tilde{\mathbf{x}}_{m}(k)+v_{m}(k)
\end{gathered}
$$

with the state transition matrix $\tilde{\mathbf{F}}$ and the vector $\mathbf{h}$ which relates states to measurements

$$
\tilde{\mathbf{F}}=\left(\begin{array}{ccc}
1 & T & \frac{1}{2} T^{2} \\
0 & 1 & \mathrm{~T} \\
0 & 0 & 1
\end{array}\right) \text { and } \mathbf{h}=\left[\begin{array}{lll}
1 & 0 & 0
\end{array}\right]
$$

$\mathbf{w}_{m}(k)$ is the process noise vector with zero mean and covariance matrix [5].

$$
\mathbf{Q}_{m}(k)=\sigma_{w}^{2}\left(\begin{array}{ccc}
T^{4} / 4 & T^{3} / 2 & T^{2} / 2 \\
T^{3} / 2 & T^{2} & T \\
T^{2} / 2 & T & 1
\end{array}\right)
$$

$v_{m}(k)$ is a zero-mean noise with variance $\sigma_{v}^{2}$, uncorrelated with $\mathbf{w}_{m}(k)$. Theoretically, the variance $\sigma_{v}^{2}$ is basically de- termined by the estimation error of the MUSIC algorithm. Because angle-of-arrival is estimated via MUSIC and furnished to KF as a measurement data in lieu of sensor data directly used in EKPF, the state vector of each target can be processed in parallel.

- Initially, the MUSIC algorithm is used twice to obtain two angle estimates $\theta_{-1}$ and $\theta_{0}$ at $k=0$. Then the initial estimate of angle and angle speed can be obtained as $\hat{\theta}_{0}=\theta_{0}$ and $\hat{\dot{\theta}}=\left(\theta_{0}-\theta_{-1}\right) / T$; namely, the initial state vector is $\hat{\mathbf{x}}(0)=\left[\begin{array}{lll}\hat{\theta}_{0} & \hat{\dot{\theta}}_{0} & 0\end{array}\right]^{T}$ and its covariance matrix is given by [5]

$$
\mathbf{P}_{m}(0 \mid 0)=\sigma_{v}^{2}\left(\begin{array}{ccc}
1 & 1 / T & 0 \\
1 / T & 2 / T^{2} & 0 \\
0 & 0 & 0
\end{array}\right)
$$

- For $k=1,2, \ldots$, do the following.

(a) Signal subspace determination via SW-OPAST

Apply the SW-OPAST algorithm to obtain the signal subspace matrix W( $k)$ recursively using $K$ snapshots of sensor data.

(b) Prediction of DOA angles

Obtain the predicted estimate $\hat{\mathbf{x}}_{m}(k \mid k-1)$ of the state vector $\tilde{\mathbf{x}}_{m}(k)$ from the existing estimates $\hat{\mathbf{x}}_{m}(k-1 \mid k-1)$ available at time $k T$ and their covariance matrices $\mathbf{P}_{m}(k \mid k-$ 1), $m=1, \ldots, M$ by the equations

$$
\begin{gathered}
\hat{\mathbf{x}}_{m}(k \mid k-1)=\tilde{\mathbf{F}} \hat{\mathbf{x}}_{m}(k-1 \mid k-1) \\
\mathbf{P}_{m}(k \mid k-1)=\tilde{\mathbf{F}} \mathbf{P}_{m}(k-1 \mid k-1) \tilde{\mathbf{F}}^{T}+\mathbf{Q}_{m}(k)
\end{gathered}
$$

The first component of $\hat{\mathbf{x}}_{m}(k \mid k-1)$ gives the predicted angle-of-arrival $\hat{\theta}_{m}(k \mid k-1)$.

(c) DOA angles estimation via MUSIC

To maximize the spatial spectrum of the MUSIC algorithm, we obtain an estimate of DOA angles $\hat{\theta}_{m}(k)$ via the Newton method [6] (initialized with $\left.\hat{\theta}_{m}(k \mid k-1)\right)$ as

$$
\hat{\theta}_{m}(k)=\hat{\theta}_{m}(k \mid k-1)-\left.\frac{\operatorname{Re}\left[\mathbf{d}^{H}(\theta) \hat{\boldsymbol{\Pi}}(k) \mathbf{a}(\theta)\right]}{\mathbf{d}^{H}(\theta) \hat{\boldsymbol{\Pi}}(k) \mathbf{d}(\theta)}\right|_{\theta=\hat{\theta}_{m}(k \mid k-1)}
$$

where $\hat{\mathbf{\Pi}}(k)=\mathbf{I}_{L}-\mathbf{W}(k) \mathbf{W}^{H}(k)$, and $\mathbf{d}(\theta)=d \mathbf{a}(\theta) / d \theta$. It is noted that (23) is similar to (5) in the Javier's paper [5].

(d) Sifting DOA angles via a robust decision mechanism The performance of the MUSIC algorithm worsens when target locations are getting closer to each other. The reason for this degradation in performance is the direction matrix 
becomes rank deficient. If the DOA angle estimate $\hat{\theta}_{m}(k)$ obtained in Step (c) is in large error and is still used in the subsequent KF procedure, then the algorithm has a large probability of not being able to correctly track the target trajectories. Therefore, a robust decision mechanism is proposed and stated as follows.

In principle, the motion of each target is slowly changing within a time increment $T$. Therefore, a typical source trajectory can be expanded via the Taylor series up to the first three items as

$$
\theta_{m}(k) \approx \theta_{m}(k-1)+\dot{\theta}_{m}(k-1) T+\frac{1}{2} \ddot{\theta}_{m}(k-1) T^{2}
$$

$\ddot{\theta}_{m}(k-1)$ is the angle acceleration obtained by substituting $\ddot{\theta}_{m}(k-1)=\left[\dot{\theta}_{m}(k-1)-\dot{\theta}_{m}(k-2)\right] / T$ into (24). Therefore, (24) becomes

$$
\left|\theta_{m}(k)-\theta_{m}(k-1)\right| \approx\left|\frac{3}{2} \dot{\theta}_{m}(k-1)-\frac{1}{2} \dot{\theta}_{m}(k-2)\right| T
$$

wherein the angle velocities $\dot{\theta}_{m}(k-1)$ and $\dot{\theta}_{m}(k-2)$ can be provided by the state estimates of the KF at times $k-1$ and $k$-2 respectively. If $\theta_{m}(k)$ is offered by step (c) and $\theta_{m}(k-1)$ is substituted with $\theta_{m}(k \mid k-1)$ (obtained from step (b)), then (25) becomes

$$
\left|\theta_{m}(k)-\theta_{m}(k \mid k-1)\right| \leq\left|\frac{3}{2} \dot{\theta}_{m}(k-1)-\frac{1}{2} \dot{\theta}_{m}(k-2)\right| T \triangleq \delta_{k}
$$

This is because the predicted value of the angle at time $k$ is closer than the estimated angle at time $k-1$ to the true angle at time $k$, namely

$$
\left|\theta_{m}(k)-\theta_{m}(k \mid k-1)\right| \leq\left|\theta_{m}(k)-\theta_{m}(k-1)\right|
$$

(26) forms the criterion for sifting DOA angles of the targets wherein the threshold value $\delta_{k}$ is time-variant depending on the angular velocity at the present time $k-1$ and the previous time $k-2$. When the target locations are mutually close, the associated DOA angle estimates may exhibit large errors leading to the angle difference exceeding the threshold $\delta$. In this case, (26) is not satisfied and thus the angle estimate $\hat{\theta}_{m}(k)$ should be discarded and substituted with $\theta_{m}(k \mid k-1)$, and the procedure returns to Step (a) (skipping Step (e)) for the next cycle of recursive processing. However, Step (e) should not be skipped for the succeeding cycle, regardless of whether the estimated angle satisfies (26) or not. This condition is posed to prevent excessive error accumulation that would result in tracking failure. In the case that the estimated angle satisfies (26), $\hat{\theta}_{m}(k)$ is used and updated via Step (e) before the next cycle of recursive processing begins. The sifting procedure requires only addition operation, thus its computational complexity is insignificant. However, it can reduce overall computation complexity if the step (e) is skipped, as to be discussed later.

(e) Updating the estimated DOA angles

Find the estimate $\hat{\mathbf{x}}_{m}(k \mid k)$ of the state vector $\tilde{\mathbf{x}}_{m}(k)$ by

$$
\hat{\mathbf{x}}_{m}(k \mid k)=\hat{\mathbf{x}}_{m}(k \mid k-1)+\mathbf{G}_{m}(k)[\hat{\theta}(k)-\hat{\theta}(k \mid k-1)]
$$

where the matrix $\mathbf{G}_{m}(k)$ is the Kalman gain given by

$$
\mathbf{G}_{m}(k)=\mathbf{P}_{m}(k \mid k-1) \mathbf{h}^{T}\left[\mathbf{h} \mathbf{P}_{m}(k \mid k-1) \mathbf{h}^{T}+\sigma_{v}^{2}\right]^{-1}
$$

The covariance matrix of $\hat{\mathbf{x}}_{m}(k \mid k)$ is obtained as

$$
\mathbf{P}_{m}(k \mid k)=\left[\mathbf{I}-\mathbf{G}_{m}(k) \mathbf{h}\right] \mathbf{P}_{m}(k \mid k-1)
$$

\section{Computational Complexity}

EKPF requires the number of $\left(2 L^{3}+30 L M^{2}+26 M^{3}+\right.$ $\left.16 L^{2} M\right) N$ real multiplications at $K=1$, whereas the Park's algorithm [7] and the Kong's algorithm [6] require the numbers of $3 L M^{2}+\left(3 L^{2}+L M\right) K$ and $5 M^{3}+10 L M^{2}+8 L^{2} M+$ $L M K$ real multiplications respectively. Although the computational complexity of EKPF is higher than those of others, EKPF has much better tracking success rate.

It is not available to find the exact computational complexities of the proposed RKF and Javier's algorithms [5], because the number of iterations for convergence is unpredictable for the Newton method involved in both algorithms. However, RKF requires $119 M+O(L M K)$ operations if step (c) is excluded. In addition, if step (e) is skipped due to the sifting process, then the number of $56 \mathrm{M}$ real multiplications can be further reduced.

\section{SIMULATION RESULTS}

In order to demonstrate the effectiveness of the proposed algorithms, Monte Carlo simulations were performed in the following scenarios. We consider three targets emitting uncorrelated narrowband signals that impinge on a uniform linear array of 10 sensors separated by half a wavelength. The targets are tracked over an interval of $40 \mathrm{sec}$ with $T=1 \mathrm{sec}$. During each one-second interval, we consider two cases: $K=1$ (single) and $K=25$ snapshots of sensor data were generated and used. The parameters used in the system model for all algorithms to be compared are $\sigma_{v}^{2}=3, \sigma_{w}^{2}=1$, and $\sigma_{n}^{2}=3$. For comparison, the algorithms developed by particle filter [9], Park et al. [7], Ryu et al. [10], Kong and Chun [6], and Javier and Sylvie [5] were simulated. For each algorithm, 100 

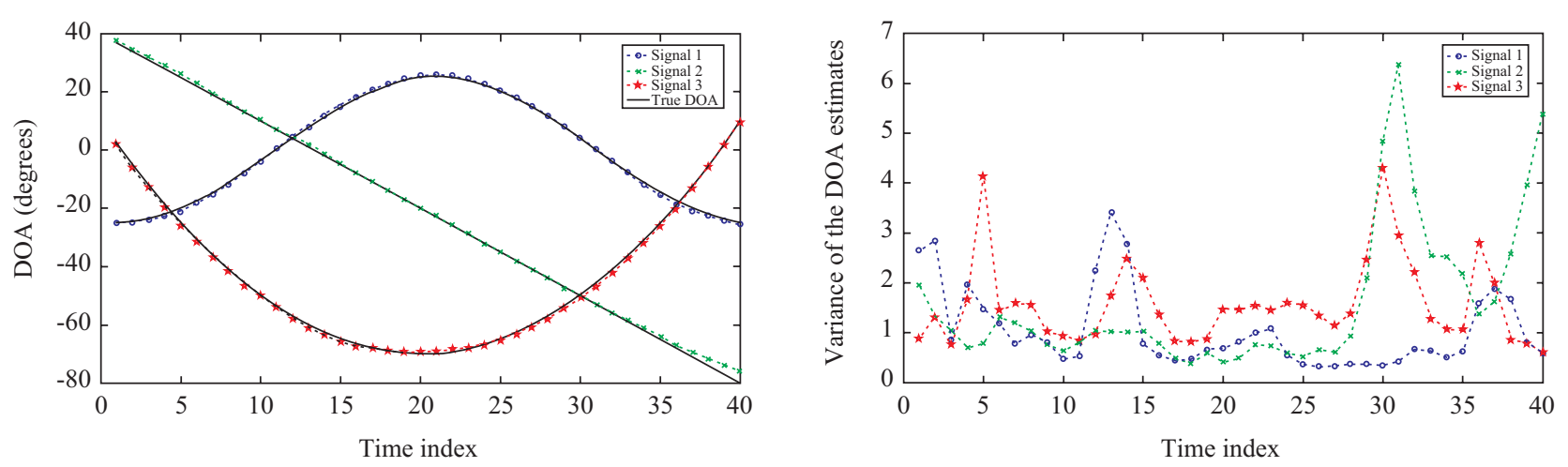

Fig. 1. The averaged tracking trajectories and variance profiles, using the proposed EKPF algorithm $(N=20)$, for three equipowered moving sources based on a single snapshot at $\mathrm{SNR}=9 \mathrm{~dB}$.

Left: averaged tracking trajectories; Right: variance profiles.

Table 1. Tracking performance at varying SNRs, $K=1$.

\begin{tabular}{|c|c|c|c|c|c|c|c|c|c|c|}
\hline \multirow{2}{*}{$\begin{array}{c}\text { SNR } \\
(\mathrm{dB})\end{array}$} & $\begin{array}{c}\text { Proposed } \\
\text { EKPF } \\
(N=20)\end{array}$ & $\begin{array}{c}\text { Particle } \\
\text { filter } \\
(N=200)\end{array}$ & Kong's & Park's & Ryu's & $\begin{array}{c}\text { Proposed } \\
\text { EKPF } \\
(N=20)\end{array}$ & $\begin{array}{c}\text { Particle } \\
\text { filter } \\
(N=200)\end{array}$ & Kong's & Park's & Ryu's \\
\hline 0 & 9 & 0 & 2 & 0 & 0 & 10.348 & - & 5.479 & - & - \\
\hline 3 & 55 & 6 & 17 & 0 & 0 & 6.6378 & 10.0252 & 3.442 & - & - \\
\hline 6 & 83 & 50 & 28 & 0 & 0 & 2.6653 & 5.0807 & 2.8461 & - & - \\
\hline 9 & 92 & 66 & 31 & 0 & 0 & 2.0185 & 2.8115 & 2.6051 & - & - \\
\hline
\end{tabular}

Table 2. Tracking performance at varying SNRs, $K=25$.

\begin{tabular}{|c|c|c|c|c|c|c|c|c|c|c|}
\hline \multirow{2}{*}{$\begin{array}{c}\text { SNR } \\
(\mathrm{dB})\end{array}$} & $\begin{array}{c}\text { Proposed } \\
\text { EKPF } \\
(N=20)\end{array}$ & $\begin{array}{c}\text { Particle } \\
\text { filter } \\
(N=200)\end{array}$ & Kong's & Park's & Ryu's & $\begin{array}{c}\text { Proposed } \\
\text { EKPF } \\
(N=20)\end{array}$ & $\begin{array}{c}\text { Particle } \\
\text { filter } \\
(N=200)\end{array}$ & Kong's & Park's & Ryu's \\
\hline 0 & 11 & 0 & 2 & 0 & 0 & 10.9989 & - & 5.58163 & - & - \\
\hline 3 & 69 & 6 & 13 & 11 & 0 & 6.0427 & 10.3463 & 6.1662 & 9.2793 & - \\
\hline 6 & 90 & 49 & 24 & 37 & 6 & 3.5138 & 5.846 & 5.7331 & 7.8439 & 18.707 \\
\hline 9 & 96 & 71 & 30 & 51 & 10 & 3.0254 & 3.6073 & 5.151 & 6.6368 & 17.4478 \\
\hline
\end{tabular}

Monte Carlo simulations of a scenario where three sources cross at different time instants were performed. The signal-tonoise ratio is defined as $\mathrm{SNR}=10 \log \left(\mathrm{s} / \sigma_{n}^{2}\right)$ in $\mathrm{dB}$, where $s$ is the signal power.

Figure 1 depicts the mean trajectories and variance profiles that are obtained by averaging over all respective successful tracking results out of 100 independent runs using the proposed EKPF algorithm (with $N=20$ ) for three equipowered sources based on a single snapshot of data at $\mathrm{SNR}=9 \mathrm{~dB}$. A success state is declared if the angle estimated is deviated from the true angle by less than $5^{\circ}$, which corresponds to about a half 3-dB beam width. The proposed EKPF algorithm can accurately track each target trajectory at this level of SNR. As expected, there is a tendency that larger variance exhibits when targets are mutually closed.
Tables 1 and 2, respectively, exhibit the tracking performances of the three moving targets at varying SNRs for $K=1$ and $K=25$ snapshots of sensor data. The proposed EKPF algorithm using a smaller number of particles $(N=20)$, can significantly increase the percentage of tracking success as compared to the particle filter with $N=200$, Kong's (EKF) algorithm, and other KF-based algorithms. It should be noted that only the proposed, particle filter, and Kong's algorithms can successfully track target trajectories with a single snapshot of data. In computing the mean square error (MSE), only the successful tracking results are taken into account. While competing with the Kong's algorithm in accuracy, the proposed algorithm exhibits much lower MSE values than the other algorithms in comparison. 

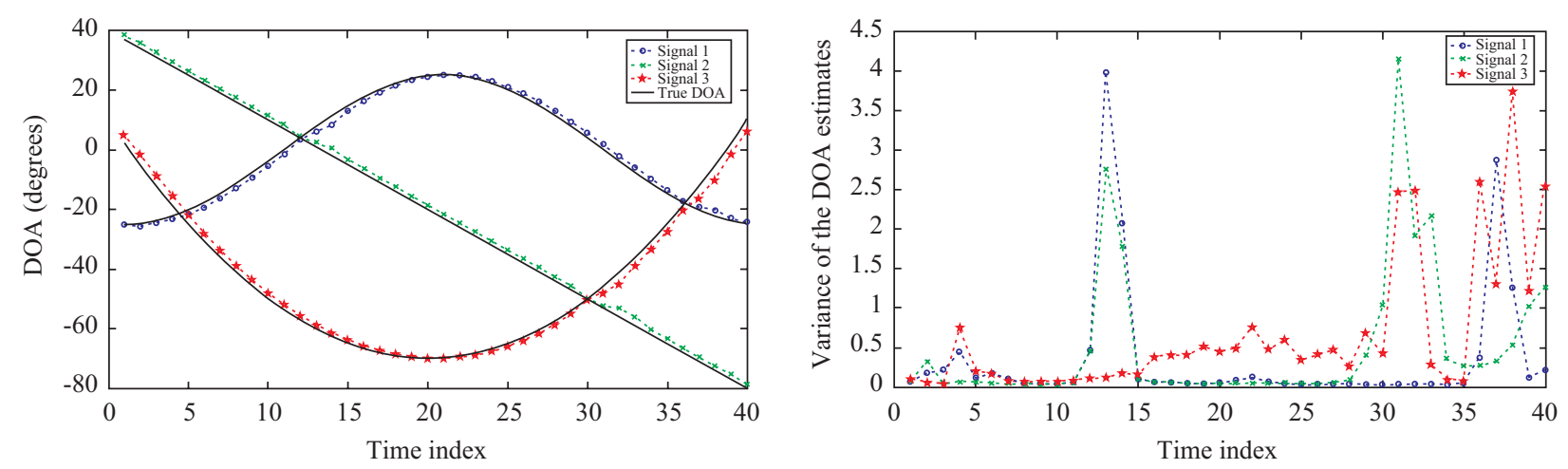

Fig. 2. The averaged tracking trajectories and variance profiles, using the proposed RKF algorithm, for three equipowered moving sources based on 25 snapshots at $\mathrm{SNR}=6 \mathrm{~dB}$.

Left: averaged tracking trajectories; Right: variance profiles.

Table 3. Tracking performance at varying SNRs, $K=25$.

\begin{tabular}{|c|c|c|c|c|c|c|}
\hline \multirow{2}{*}{$\begin{array}{c}\text { SNR } \\
(\mathrm{dB})\end{array}$} & \multicolumn{3}{|c|}{ Tracking success rate (\%) } & \multicolumn{2}{c|}{ MSE (deg $\left.{ }^{2}\right)$} \\
\cline { 2 - 7 } & $\begin{array}{c}\text { Proposed } \\
\text { RKF }\end{array}$ & $\begin{array}{c}\text { Javier's with the } \\
\text { decision mechanism }\end{array}$ & Javier's & $\begin{array}{c}\text { Proposed } \\
\text { RKF }\end{array}$ & $\begin{array}{c}\text { Javier's with the } \\
\text { decision mechanism }\end{array}$ & Javier's \\
\hline-3 & 55 & 41 & 1 & 7.3622 & 6.1263 & 4.1111 \\
\hline 0 & 72 & 70 & 15 & 5.1599 & 3.7915 & 2.3723 \\
\hline 3 & 93 & 90 & 51 & 4.2002 & 2.6361 & 1.9561 \\
\hline 6 & 99 & 98 & 93 & 3.6297 & 2.4073 & 1.7203 \\
\hline
\end{tabular}

Figure 2 depicts the mean trajectories and variance profiles that are obtained by averaging over all respective successful tracking results out of 100 independent runs using the proposed RKF algorithm for three equipowered sources based on 25 snapshots of data at SNR $=6 \mathrm{~dB}$. Similarly, the proposed $\mathrm{RKF}$ algorithm can accurately track each target trajectory at this level of SNR. As expected, there is a tendency that larger variance exhibits when targets are mutually closed.

Table 3 exhibits the tracking performances of the three moving targets at varying SNRs for $K=25$ snapshots of sensor data. It is evident that Javier's algorithm with the proposed decision mechanism incorporated and the proposed RKF algorithm can significantly increase the tracking success rate at the price of a degraded level of accuracy. Moreover, the proposed RKF algorithm has the best tracking success rate among all algorithms under comparison.

\section{CONCLUSIONS}

We have presented two novel tracking algorithms using a single snapshot and multiple snapshots of data measured from a sensor array. The RKF algorithm is suitable only for the case of multiple snapshots of data, whereas the EKPF algorithm is capable for the case of a single snapshot of data within a time increment. The EKPF algorithm allows us to improve the tracking success rate over the particle filter and the EKF. As illustrated in the simulation results, the proposed EKPF algorithm offers the best tracking success rate as compared with other methods at both single and multiple snapshots of data. While competing with the Kong's algorithm in accuracy, the proposed algorithm exhibits much lower MSE values than the other algorithms in comparison.

For the case of multiple snapshots within a time increment, a robust decision mechanism is presented and used in conjunction with the Kalman filter, leading to a novel algorithm for tracking the angles-of-arrival of multiple moving sources. The proposed RKF algorithm utilizes the angles-of-arrival obtained from the MUSIC algorithm as the measurement data for the Kalman filter. Prior to updating the estimated anglesof-arrival, these angle estimates are sifted via the proposed robust decision mechanism to prevent large error propagation. As illustrated in the simulation results, the proposed RKF algorithm offers the best tracking success rate at the price of larger MSE value, as compared with Javier's algorithm especially at low SNRs.

\section{ACKNOWLEDGMENTS}

This work was supported in part by National Science Council of TAIWAN under contracts NSC 98-2221-E-019-066- and NSC 96-2221-E-022-016-. The authors would like to thank unknown reviewers for invaluable comments and suggestions. 


\section{REFERENCES}

1. Badeau, R., Abed-Meraim, K., Richard, G., and David, B., "Sliding window orthonormal PAST algorithm," Proceeding of IEEE ICASSP'03, pp. V-261-V-264 (2003).

2. Bar-Shalom, Y. and Fortmann, T. E., Tracking and Data Association, New York, Academic (1988).

3. Chang, S. H., Hou, S. Y., Chang, S. C., and Hung, H. S., "Underwater wideband signal tracking based on predictive angle tracking algorithm," Journal of Marine Science and Technology, Vol. 13, No. 1, pp. 46-53, March (2005).

4. Gordon, N., Salmond, D., and Smith, A., "Novel approach to nonlinear/nonGaussian Bayesian state estimation," Radar and Signal Processing, IEE Proceedings-F, Vol. 140, No. 2, pp. 107-113 (1993).

5. Javier, S. A. and Sylvie, M., "An efficient PASTd-algorithm implementation for multiple direction of arrival tracking," IEEE Transaction on Signal Processing, Vol. 47, No. 8, pp. 2321-2324 (1999).

6. Kong, D. and Chun, J., "A fast DOA tracking algorithm based on the extended Kalman filter," Proceeding of the IEEE NAECON Conference, pp. 235-238 (2000).

7. Park, S. B., Ryu, C. S., and Lee, K. K., "Multiple target tracking algorithm using predicted angles," IEEE Transaction on Aerospace and Electronic Systems, Vol. 30, No. 2, pp. 643-648 (1994)

8. Rao, C. R., Sastry, C. R., and Zhou, B., "Tracking the direction of arrival of multiple moving targets," IEEE Transaction on Signal Processing, Vol. 42, No. 5, pp. 1133-1144 (1994).

9. Ristic, B., Arulampalam, S., and Gordon, N., Beyond the Kalman Filter: Particle Filters for Tracking Applications, Artech House, Norwell, Massachusetts (2004).

10. Ryu, C. S., Lee, J. S., and Lee, K. K., "Multiple target angle tracking algorithm with efficient equation for angular innovation," IEE Electronics Letters, Vol. 38, No. 10, pp. 483-484 (2002).

11. Ryu, C. S., Lee, S. H., and Lee, K. K., "Multiple target angle tracking algorithm using innovation extracted from signal subspace," IEE Electronics Letters, Vol. 35, No. 18, pp. 1520-1522 (1999).

12. Schmidt, R. O., "Multiple emitter location and signal parameter estimation," IEEE Transaction on Antennas and Propagation, Vol. 34, No. 3, pp. 276-280 (1986)

13. Simon, D., Optimal State Estimation, John Wiley \& Sons, Inc. (2006).

14. Sword, C. K., Simaan, M., and Kamen, W. W., "Multiple target angle tracking using sensor array output," IEEE Transaction on Aerospace and Electronic Systems, Vol. 26, No. 3, pp. 367-372 (1990).

15. Yang, B., "Projection approximation subspace tracking," IEEE Transaction on Signal Processing, Vol. 43, pp. 95-107 (1995).

16. Yang, J. and Kaveh, M., "Adaptive eigensubspace algorithms for direction or frequency estimation and tracking," IEEE Transaction on Acoustics Speech and Signal Processing, Vol. 36, pp. 241-251 (1988). 